\title{
SOBOLEV AND QUASICONFORMAL EXTENSION DOMAINS
}

\author{
MANOUCHEHR GHAMSARI
}

(Communicated by Clifford J. Earle)

\begin{abstract}
A domain $D \subset \mathbb{R}^{n}$ has the quasiconformal extension property if each quasiconformal self-map of $D$ extends to a quasiconformal self-map of $\mathbb{R}^{n} ; D$ has the Sobolev extension property if there is a bounded linear operator $\Lambda: W^{1, n}(D) \rightarrow W^{1, n}\left(\mathbb{R}^{n}\right)$. We consider the relation between the above extension properties for $n \geq 3$. We show that for domains quasiconformally equivalent to a ball the quasiconformal extension property implies the Sobolev extension property. We show that this is not true in general. Next the Sobolev extension property does not imply the extension property for quasiconformal maps which extend as homeomorphisms. Finally if $G \subset \mathbb{R}^{2}$ and if $D=G \times \mathbb{R}$ is quasiconformally equivalent to a ball, then $D$ has the quasiconformal extension property if and only if $D$ is a quasiball.
\end{abstract}

\section{INTRODUCTION}

In the study of $K$-quasiconformal self-maps of domains $D \subset \mathbb{R}^{n}$, a commonly examined question is when each such map extends to a global $K^{\prime}$ quasiconformal self-map of $\mathbb{R}^{n}, K^{\prime}=K^{\prime}(K)$. For simply connected domains $D \subset \mathbb{R}^{2}$, the problem has been solved; $D$ has the quasiconformal extension property if and only if $D$ is a quasidisk, and a similar result for finitely connected domains is due to Gehring and Hag [GH, Theorem 3.10].

In higher dimensions the problem is much harder even for domains which are quasiconformally equivalent to $B^{n}$. The reason is that in this case we have only fragmentary information about the domains which can be mapped by a quasiconformal map onto a ball in $\mathbb{R}^{n}$.

The domain $D$ has the Sobolev extension property if there is a bounded linear extension operator $\Lambda: W^{1, n}(D) \rightarrow W^{1, n}\left(\mathbb{R}^{n}\right)$. Jones established that a simply connected bounded domain $G \subset \mathbb{R}^{2}$ has this property if and only if $G$ is a quasidisk [J, Theorem C]. In particular, he proved that the so-called $(\varepsilon, \delta)$ domains have the Sobolev extension property [J, Theorem 2]. The $(\varepsilon, \delta)$ domains contain uniform domains as a proper subclass; definitions are recalled in $\S 2$.

As quasiconformal maps $f: D \rightarrow \overline{\mathbb{R}}^{n}$ are in $W_{\text {loc }}^{1, n}(D)$, it is natural to ask how the quasiconformal extension property and the Sobolev extension property are related for domains $D$ in $\overline{\mathbb{R}}^{n}$. In [GH, Example 4.4], Gehring and Hag

Received by the editors December 9, 1991 and, in revised form, March 27, 1992.

1991 Mathematics Subject Classification. Primary 30C65; Secondary 47A20. 
constructed a quasiconformal self-map of a uniform domain $D$ which carries a flat boundary point to a nonflat one. Such a map does not have an extension even to a homeomorphism of $\mathbb{R}^{n}$; there the obstruction to quasiconformal extension is a topological one. It remains to see whether the Sobolev extension property implies the quasiconformal extension property for those quasiconformal self-maps of $D$ which are the restriction to $D$ of self-homeomorphisms of $\overline{\mathbb{R}}^{n}$. We show that quasiconformal self-maps of $(\varepsilon, \sigma)$ domains need not extend even when they extend as self-homeomorphisms of $\mathbb{R}^{n}$.

This paper is organized as follows. Preliminaries are given in $\S 2$; in $\S 3$ we consider the quasiconformal extension property for domains quasiconformally equivalent to the unit ball and show that the quasiconformal extension property implies the Sobolev extension property for such domains. In $\S 4$ we show that $B^{2} \times \mathbb{R}$ does not have the extension property for quasiconformal self-maps which are the restriction to $B^{2} \times \mathbb{R}$ of self-homeomorphisms of $\mathbb{R}^{3}$, and thus the Sobolev extension property does not imply the quasiconformal extension property even for such maps. We construct a bounded Jordan domain in $\S 5$ which has the quasiconformal but not the Sobolev extension property. In $\S 6$ we characterize cylindrical domains in $\mathbb{R}^{3}$ which are quasiconformally equivalent to $B^{3}$ and have the quasiconformal extension property.

Some of the results in this paper constitute a part of my thesis. I would like to thank my advisor, Professor F. W. Gehring, for suggesting the problem and for his support during my study at the University of Michigan.

\section{Preliminaries}

2.1. Extension property. We say that a domain $D \subset \overline{\mathbb{R}}^{n}$ has the extension property for a class of mappings $\mathscr{F}$ if each self-map $f$ of $D$ in $\mathscr{F}$ has an extension to a self-map $\tilde{f}$ of $\overline{\mathbb{R}}^{n}$ or $\mathbb{R}^{n}$ in $\mathscr{F}$. We are interested in the above extension property for the class of bilipschitz, quasisymmetric, or quasiconformal maps.

An embedding $f: E \subset \mathbb{R}^{n} \rightarrow \mathbb{R}^{n}$ is L-bilipschitz if for each pair of points $x, y \in E$

$$
|x-y| / L \leq|f(x)-f(y)| \leq L|x-y|, \quad L \geq 1 .
$$

An embedding $f: X \subset \mathbb{R}^{k} \rightarrow \mathbb{R}^{n}$ is said to be $\eta$-quasisymmetric if there is a homeomorphism $\eta:[0, \infty) \rightarrow[0, \infty]$ such that

$$
|x-a| \leq t|y-a| \Rightarrow|f(x)-f(a)| \leq \eta(t)|f(y)-f(a)|
$$

for all $a, x, y \in X$ and all $t>0$. One can always assume that $\eta(t) \geq t$. See [TV1] for properties of quasisymmetric maps.

Let $D$ and $D^{\prime}$ be domains in $\overline{\mathbb{R}}^{n}$. A homeomorphism $f: D \rightarrow D^{\prime}$ is $K$-quasiconformal if for each curve family $\Gamma$ in $D, M(\Gamma) / K \leq M(f(\Gamma)) \leq$ $K M(\Gamma)$, where $M(\Gamma)$ is the modulus of $\Gamma$. For basic information about quasiconformal maps see [V1].

The following result relates the extension properties in the various classes of maps indicated above.

2.2. Theorem [TV2, Theorem 2.19]. If $n \neq 4$ and if $f: E \subset \mathbb{R}^{n} \rightarrow \mathbb{R}^{n}$ is $a$ bilipschitz map, then $f$ has an extension to a quasiconformal self-map of $\mathbb{R}^{n}$ if and only if $f$ has an extension to a bilipschitz self-map of $\mathbb{R}^{n}$. 
2.3. $(\varepsilon, \delta)$ domains. A domain $D \subset \mathbb{R}^{n}$ is said to be $c$-uniform if each pair of points $x$ and $y$ in $D$ can be joined by an arc $\gamma$ in $D$ such that

$$
\left\{\begin{array}{l}
l(\gamma) \leq c|x-y|, \\
\min \left(l\left(\gamma_{1}\right), l\left(\gamma_{2}\right)\right) \leq c \operatorname{dist}(z, \partial D),
\end{array}\right.
$$

for each $z \in \gamma$, where $\gamma_{1}, \gamma_{2}$ are the components of $\gamma \backslash\{z\}$. Next, $D$ is an $(\varepsilon, \delta)$ domain if (2.4) holds for all $x, y$ in $D$ for which $|x-y|<\varepsilon$ and for $\delta$ in the place of $c$. A bounded $(\varepsilon, \delta)$ domain is a uniform domain; $B^{2} \times \mathbb{R}$ is an $(\varepsilon, \delta)$ domain which is not uniform.

2.5. Definition. A domain $D$ is c-quasiconvex if each pair of points $x$ and $y$ in $D$ can be joined by a path $\gamma$ in $D$ such that $l(\gamma) \leq c|x-y|$. Uniform domains are quasiconvex, and, in general, if $D$ has the Sobolev extension property, then $D$ is quasiconvex [K, Theorem 5.8]; see also [GR, $\S 6$, Theorem 3.3].

In the proof of Theorem 6.1 we use the following result due to Väisälä for cylinderical domains $D=G \times \mathbb{R}, G \subset \mathbb{R}^{2} ; D$ is quasiconformally equivalent to $B^{3}$ if and only if $G$ is an inner chordarc domain [V3].

Let $x$ be a boundary point of a domain $D$. We say that $D$ is finitely connected at $x$ if $x$ has arbitrarily small neighborhoods $U$ so that $U \cap D$ has a finite number of components; $n$-connectedness at $x$ is defined similarly. A simply connected domain $G \subset \mathbb{R}^{2}$ with locally rectifiable boundary is $c$-inner chordarc if it is finitely connected on the boundary and if, for each pair of finite boundary points $z_{1}$ and $z_{2}, \min \left(l\left(\gamma_{1}\right), l\left(\gamma_{2}\right)\right) \leq c \lambda\left(z_{1}, z_{2}\right)$. Here $\gamma_{1}$ and $\gamma_{2}$ are the components separated by $z_{1}, z_{2}$ in the boundary and $\lambda\left(z_{1}, z_{2}\right)=$ $\inf l(\beta)$, where the infimum is taken over all open $\operatorname{arcs} \beta$ in $G$ which join $z_{1}$ and $z_{2}$.

For a domain $D \subset \overline{\mathbb{R}}^{n}, \partial D, \bar{D}$, and $D^{*}=\overline{\mathbb{R}}^{n} \backslash \bar{D}$ denote the boundary, the closure, and the exterior of $D$, respectively. The ball centered at $x$ of radius $r$ is denoted by $B^{n}(x, r), S^{n-1}(x, r)$ is its boundary, and $H^{n}=\{x=$ $\left.\left(x_{1}, \ldots, x_{n}\right): x_{n}>0\right\}$. We use $l(\gamma) \in[0, \infty]$ for the length of arcs $\gamma$ in $\mathbb{R}^{n}$, denote the closed segment between a pair of points $x_{1}, x_{2} \in \mathbb{R}^{n}$ by $\left[x_{1}, x_{2}\right]$, and the closed subarc of $\gamma$ from $x_{1}$ to $x_{2}$ is denoted by $\gamma\left[x_{1}, x_{2}\right]$.

\section{EXTENSION PROPERTIES OF DOMAINS}

WHICH ARE QUASICONFORMALLY EQUIVALENT TO A BALL

3.1. Double-quasiball condition. We say that a bounded Jordan domain $D \subset$ $\mathbb{R}^{n}$ satisfies the $(c, K)$-double-quasiball condition if for each $b \in \partial D \backslash\{\infty\}$ there exist $K$-quasiballs $Q \subset D, Q^{\prime} \subset D^{*}$ so that

(a) $b \in \partial Q \cap \partial Q^{\prime}$;

(b) $\min \left(\operatorname{diam}(Q), \operatorname{diam}\left(Q^{\prime}\right)\right) \geq(1 / c) \operatorname{diam}(D)$.

If $\infty \in \partial D$, then (b) is replaced by

$\left(\mathrm{b}^{\prime}\right)$ for each $r>0$ there exist $Q, Q^{\prime}$ such that $\min \left(\operatorname{diam}(Q), \operatorname{diam}\left(Q^{\prime}\right)\right)$ $\geq r$.

3.2. Theorem. Suppose that $D$ is homeomorphic to $B^{n}$ via a $K$-quasiconformal map $f$. If $D$ has the quasiconformal extension property, then

(a) $D$ is a b-uniform domain, $b=b(K, n)$;

(b) D satisfies a double-quasiball condition. 
Proof. (a) First, $D$ is a Jordan domain and $f$ has a homeomorphic extension to the closures [N2, Theorem 9.6]. After performing preliminary Möbius transformations we may assume that $\infty \in \partial D, f: H^{n} \rightarrow D$, and $f$ fixes the point at $\infty$.

We show that $f \mid \partial H^{n}$ is quasisymmetric. Choose $x_{0}, x_{1}, x_{2} \in \partial H^{n}$ such that $\left|x_{1}-x_{0}\right|=\left|x_{2}-x_{0}\right|$, and let $\phi$ be the reflection in the bisector of $\left[x_{1}, x_{2}\right]$. Then $g=f \circ \phi \circ f^{-1}$ is a $K^{2}$-quasiconformal self-map of $D$. There is a $K_{1}$ quasiconformal self-map $g_{1}$ of $\mathbb{R}^{n}$ which agrees with $g$ on $D, K_{1}=K_{1}(K)$, by the extension property of $D$. We conclude that $g_{1}$ and hence $g$ are $\eta_{1}$ quasisymmetric, $\eta_{1}=\eta_{1}(K, n)$, and hence

$$
\frac{\left|y_{2}-y_{0}\right|}{\left|y_{1}-y_{0}\right|} \leq \eta_{1}\left(\frac{\left|y_{1}-y_{0}\right|}{\left|y_{2}-y_{0}\right|}\right)=\eta_{1}(t),
$$

where $y_{j}=f\left(x_{j}\right)$ for $j=0,1,2$.

Next if $t<\eta_{1}^{-1}(1)$, then $\eta_{1}(t)<1$ and by $t \leq \eta_{1}(1)$ we obtain $t \eta_{1}(t)<1$, contradicting the above inequality. Thus $t \geq \eta_{1}^{-1}(1)$, and by symmetry $1 / t \geq$ $\eta_{1}^{-1}(1)$. Hence

$$
\frac{1}{H} \leq \frac{\left|f\left(x_{1}\right)-f\left(x_{0}\right)\right|}{\left|f\left(x_{2}\right)-f\left(x_{0}\right)\right|} \leq H, \quad H=\left(\eta_{1}^{-1}(1)\right)^{-1},
$$

whenever $\left|x_{1}-x_{0}\right|=\left|x_{2}-x_{0}\right|$. Therefore $f \mid H^{n}$ is weakly $H$-quasisymmetric and hence $\eta^{\prime}$-quasisymmetric, $\eta^{\prime}=\eta^{\prime}(K, n)$. By [V4, Theorem 5.6] both $D$ and $D^{*}$ are uniform.

(b) We assume that $\infty \in \partial D$ and $f: H^{n} \rightarrow D$ is a quasiconformal map fixing $\infty$. Then by (a) $f$ is $\eta$-quasisymmetric, $D$ is uniform, and $\eta=\eta(K, n)$. Fix $x \in \partial D, r>0$, choose $x_{1} \in \partial D$ so that $\left|x-x_{1}\right|=r$, and let $\alpha$ be a uniform arc joining $x$ and $x_{1}$ in $D$. If $x_{0}$ is the midpoint of $\alpha$ then

$$
\operatorname{dist}\left(x_{0}, \partial D\right) \geq r / b=r^{\prime}
$$

and there is a point $x^{\prime} \in \partial D$ such that $\left|x_{0}-x^{\prime}\right|=r^{\prime}$. Hence

$$
\left|x-x^{\prime}\right| \leq\left|x-x_{0}\right|+\left|x_{0}-x^{\prime}\right| \leq(1+b) r .
$$

Let $y^{\prime}=f^{-1}\left(x^{\prime}\right), y=f^{-1}(x)$, and let $\psi$ be the translation of $\mathbb{R}^{n}$ sending $y^{\prime}$ to $y$. Then $h=f \circ \psi \circ f^{-1}$ is a $K^{2}$-quasiconformal self-map of $D$, and it has an extension to a $K^{\prime}$-quasiconformal map $h$ of $\mathbb{R}^{n}$. Therefore $h$ is $\eta$-quasisymmetric and

$$
\frac{\left|h(x)-h\left(x^{\prime}\right)\right|}{\left|h\left(x^{\prime}\right)-h\left(x_{0}\right)\right|} \leq \eta\left(\frac{\left|x-x^{\prime}\right|}{\left|x_{0}-x^{\prime}\right|}\right) \leq \eta(b(b+1))=H .
$$

Therefore

$$
\begin{aligned}
\left|h\left(x^{\prime}\right)-h\left(x_{0}\right)\right| & \geq\left|h(x)-h\left(x^{\prime}\right)\right| / H=\left|f(\psi(y))-f\left(\psi\left(y^{\prime}\right)\right)\right| / H \\
& \geq\left|f(y)-f\left(y^{\prime}\right)\right| / H \eta(1)=\left|x-x^{\prime}\right| / H \eta(1)=r / M,
\end{aligned}
$$

because $f$ is $\eta$-quasisymmetric, $M=M(K, n)$.

If $Q=h\left(B^{n}\left(x_{0}, r\right)\right)$, then $Q$ is a $K^{\prime}$-quasiball in $D, x \in \partial Q$, and $\operatorname{diam}(Q) \geq r / M$. Similarly, we obtain a quasiball $Q^{\prime}$ in $D^{*}$ with the desired properties. 


\section{THE SOBOLEV EXTENSION PROPERTY}

DOES NOT IMPLY THE QUASICONFORMAL EXTENSION PROPERTY

The cylinder $B^{2} \times \mathbb{R}$ is a simple example of a nonuniform $(\varepsilon, \delta)$ domain which is quasiconformally equivalent to a ball. Of course, this domain does not have the quasiconformal extension property because it is 2-connected at $\infty$, and any homeomorphism for which the cluster set of $\infty$ contains two distinct points does not extend even as a homeomorphism. We consider the extension problem for quasiconformal maps which do extend as homeomorphisms.

4.1. Theorem. The cylinder $B^{2} \times \mathbb{R}$ does not have the extension property for the class of quasiconformal maps fixing $\infty$.

The proof starts at $\S 4.2$ and ends at $\S 4.4$ and is based on existence of a family of "screw-type" self-maps of $B^{2} \times \mathbb{R}$ with no extension to a quasiconformal selfmap of $\mathbb{R}^{3}$.

I wish to thank Professor J. Väisälä for his observation that the following screw-type maps can be used to show that $B^{2} \times \mathbb{R}$ does not have the extension property for quasiconformal maps.

4.2. Screw-type mappings. Let $(r, \theta, t)$ denote the cylindrical coordinates of a point $x=(z, t) \in \mathbb{R}^{3}$. Then the homeomorphism $f_{c}: B^{2} \times \mathbb{R} \rightarrow B^{2} \times \mathbb{R}$, where $f_{c}(r, \theta, t)=(r, \theta+c t, t)$, is said to be a screw-type mapping. We show that $f_{c}$ is bilipschitz in $B^{2} \times \mathbb{R}$. Let $x=(r, \theta, t), x^{\prime}=\left(r^{\prime}, \theta^{\prime}, t^{\prime}\right)$ be the cylinderical coordinates of the points $x$ and $x^{\prime}$ and let $\phi=\theta+c t, \phi^{\prime}=\theta^{\prime}+c t^{\prime}$. Then

$$
\begin{aligned}
\left|f_{c}(x)-f_{c}\left(x^{\prime}\right)\right|^{2} & =\left|r \cos \phi-r^{\prime} \cos \phi^{\prime}\right|^{2}+\left|r \sin \phi-r^{\prime} \sin \phi^{\prime}\right|^{2}+\left|t-t^{\prime}\right|^{2} \\
& =r^{2}+r^{\prime 2}-2 r r^{\prime} \cos \left(\phi-\phi^{\prime}\right)+\left|t-t^{\prime}\right|^{2} \\
& =\left|x-x^{\prime}\right|^{2}+2 r r^{\prime}\left[-\cos \left(\phi-\phi^{\prime}\right)+\cos \left(\theta-\theta^{\prime}\right)\right] .
\end{aligned}
$$

Next, using the inequality

$$
|\cos (a)-\cos (b)|=|a-b||\sin (b+h(a-b))| \leq|a-b|(|\sin (b)|+|a-b|),
$$

we obtain

$$
\left|\cos \left(\phi-\phi^{\prime}\right)-\cos \left(\theta-\theta^{\prime}\right)\right| \leq|c|\left|t-t^{\prime}\right|\left\{\left|\sin \left(\theta-\theta^{\prime}\right)\right|+|c|\left|t-t^{\prime}\right|\right\} .
$$

In the triangle with vertices at $0, z, z^{\prime}$,

$$
\frac{\sin \left(\theta-\theta^{\prime}\right)}{\left|z-z^{\prime}\right|}=\frac{\sin \left(\angle 0 z^{\prime} z\right)}{r}
$$

by the "sine law", whence

$$
\begin{aligned}
\left|f_{c}(x)-f_{c}\left(x^{\prime}\right)\right|^{2} & \leq\left|x-x^{\prime}\right|^{2}+2 r^{\prime}|c|\left|t-t^{\prime}\right|\left[\left|z-z^{\prime}\right|+|c|\left|t-t^{\prime}\right|\right] \\
& \leq\left|x-x^{\prime}\right|^{2}+2|c|(1+|c|)\left|x-x^{\prime}\right|^{2}=c^{\prime 2}\left|x-x^{\prime}\right|^{2} .
\end{aligned}
$$

Therefore $f_{c}$ is $c^{\prime}$-lipschitz and since $f_{-c}=f_{c}^{-1}, f_{c}^{-1}$ is also $c^{\prime}$-lipschitz. As a result $f_{c}$ is $c^{\prime}$-bilipschitz and hence $K$-quasiconformal,

$$
K\left(f_{c}\right)=c^{\prime 4} \rightarrow 1 \quad \text { as } c \rightarrow 0 .
$$

For the rest of this section and $\S 5$ we let $L_{r}=\{(r, 0, t): t \in \mathbb{R}\}$. For a path $\beta:[0,1] \rightarrow \mathbb{R}^{2}, \beta(s) \neq 0$, the unique continuous function $\varphi(s)$,

$$
\varphi(s)=\arg (\beta(s)) \text { for } s \in[0,1], \varphi(0) \in[0,2 \pi),
$$

will be denoted by $\arg (\beta)$. 
4.3. Lemma. The cylinder $B^{2} \times \mathbb{R}$ does not have the bilipshitz extension property.

Proof. We show that the screw-type maps defined above do not have bilipschitz extensions to $\mathbb{R}^{3}$. Set $f=f_{1}$, where $f_{c}$ is as in $\S 4.2$, and suppose that $\tilde{f}$ is an $L$-bilipschitz self-map of $\mathbb{R}^{3}$ which agrees with $f$ on $B^{2} \times \mathbb{R}$. Let $r=$ $2 L^{2}+1$, fix $n>4 L\left(2 L^{2}+1\right)$, and consider the rectangle with vertices at $(1,0,0),(r, 0,0),(r, 0, n),(1,0, n)$. Suppose that $\gamma:[0,1] \rightarrow \mathbb{R}^{3}$ is a parametrization of the rectangle and that $\gamma(0)=\gamma(1)=(1,0,0), \gamma\left(s_{1}\right)=$ $(r, 0,0), \gamma\left(s_{2}\right)=(r, 0, n)$, and $\gamma\left(s_{3}\right)=(1,0, n)$. Then $\beta(s)=P \circ \tilde{f} \circ \gamma(s)$ is a closed curve in $\mathbb{R}^{2}$ where $P$ is the projection onto the plane $t=0$.

We first show that $\arg \beta(1)-\arg \beta(0)=0$. For $t \in I=[0,1]$, let $H(t, s)=$ $(1-t) \gamma(s)+t(1,0,0)$, let $H^{\prime}=\tilde{f} \circ H$, and let $H^{\prime \prime}=P \circ \tilde{f} \circ H$. Then $H, H^{\prime}: I \times I \rightarrow \mathbb{R}^{3}$ are homotopies in $\mathbb{R}^{3} \backslash L_{0}$ and $H^{\prime \prime}$ is a homotopy in $\mathbb{R}^{2} \backslash\{0\}$. But $H^{\prime \prime}(0, s)=\beta(s)$ and $H^{\prime \prime}(1, s)=P \circ f(1,0,0)$; hence, $\beta$ is homotopic to a point in $\mathbb{R}^{2} \backslash\{0\}$ and $\arg \beta(1)-\arg \beta(0)=0$. Therefore

$$
\left|\arg \beta\left(s_{3}\right)-\arg \beta(0)\right|=\left|\arg \beta\left(s_{3}\right)-\arg \beta(1)\right|=n .
$$

We show that the above relation leads to a contradiction. If $x \in \beta\left[s_{1}, s_{2}\right]$, then

$$
\operatorname{dist}\left(\tilde{f}(x), B^{2} \times \mathbb{R}\right) \geq \frac{\operatorname{dist}\left(x, B^{2} \times \mathbb{R}\right)}{L}=\frac{r-1}{L}=2 L
$$

because $\tilde{f}$ is $L$-bilipschitz. Hence

$$
\left|\arg \beta\left(s_{1}\right)-\arg \beta\left(s_{2}\right)\right| \leq \frac{l\left(\beta\left[s_{1}, s_{2}\right]\right)}{\operatorname{dist}(0, \beta)} \leq \frac{n L}{2 L}=\frac{n}{2} .
$$

In addition, $l\left(\beta\left[0, s_{1}\right]\right) \leq l\left(\tilde{f}\left(\gamma\left[0, s_{1}\right]\right)\right) \leq L(r-1)<n / 4$. Since $\operatorname{dist}(0, \beta) \geq$ 1 , we obtain

$$
\left|\arg \beta\left(s_{1}\right)-\arg \beta(0)\right|<n / 4 \text { and }\left|\arg \beta\left(s_{3}\right)-\arg \beta\left(s_{2}\right)\right|<n / 4 .
$$

Combining the above inequalities, we obtain $\left|\arg \beta\left(s_{3}\right)-\arg \beta(0)\right|<n / 4+n / 2+$ $n / 4=n$, which is impossible.

4.4. Completion of the proof of Theorem 4.1. Let $f_{1}$ be as in $\S 4.2$. Then $f_{1}$ is a bilipschitz self-map of $B^{2} \times \mathbb{R}$ and Lemma 4.3 shows that $f_{1}$ does not extend to a bilipschitz self-map of $\mathbb{R}^{3}$. Therefore it does not have an extension to a quasiconformal self-map of $\mathbb{R}^{3}$ by [TV2, Theorem 2.19].

One can replace $f_{c}$ for $f_{1}$ in the proof of Lemma 4.3 and obtain the following result.

4.5. Theorem. For each $\varepsilon>0, K=1+\varepsilon$, the cylinder $B \times \mathbb{R}$ does not have the extension property for the class of $K$-quasiconformal maps which have a homeomorphic extension to $\mathbb{R}^{n}$.

\section{THE QUASICONFORMAL EXTENSION PROPERTY DOES NOT IMPLY THE SOBOLEV EXTENSION PROPERTY}

In this section we construct a bounded domain $G$ such that $D=G \times \mathbb{R}$ has the quasiconformal extension property. We then map $D$ onto a bounded domain $D^{\prime}$ by a Möbius map. The domain $D^{\prime}$ serves as an example of a bounded Jordan domain which has the quasiconformal but not the Sobolev extension property. 


\subsection{Construction of $G$. Let}

$$
g(u)= \begin{cases}\frac{1}{2} u^{2}, & 0 \leq u \leq \frac{1}{2} \\ \frac{1}{2}(1-u)^{2}, & \frac{1}{2} \leq u \leq 1\end{cases}
$$

and let

$$
G=\left\{(u, v) \in \mathbb{R}^{2}:|v|<g(u), 0 \leq u \leq 1\right\} .
$$

Then $G$ is symmetric with respect to $v=0$. We let $\partial G_{ \pm}$denote parts of $\partial G$ for which $\pm v>0$ and define $G_{ \pm}$similarly. Except for the points $(0,0)$ and $(1,0)$ each boundary point of $G$ is contained in a 2-chordarc subarc of $\partial G$.

We next consider the domain $G \times \mathbb{R}$ and prove the following result.

5.2. Theorem. The domain $G \times \mathbb{R}$ has the quasiconformal extension property. Proof. Let $f$ be a $K$-quasiconformal map of $G \times \mathbb{R}$. We construct a $K^{\prime}$ quasiconformal map of $\mathbb{R}^{3}$ which agrees with $f$ on $G \times \mathbb{R}$.

(a) Boundary extension. We distinguish three distinct types of boundary points for $G \times \mathbb{R}$ : the points on $L_{0}=\{(0,0, t)\}$ and $L_{1}=\{(1,0, t)\}$ or the wedge points, points on $\left(\partial G_{+} \cup \partial G_{-}\right) \times \mathbb{R}$, and the point at $\infty$. For each $x_{0} \in\left(\partial G_{+} \cup \partial G_{-}\right) \times \mathbb{R}$, there is a bilipschitz self-map of $\mathbb{R}^{3}$ which maps a portion of $\partial G \times \mathbb{R}$ near $x_{0}$ into a hyperplane. This is not true for the other two types of boundary points [GV, Theorem 10.5].

Fix $x_{0} \in \partial G_{+} \times \mathbb{R}$ and let $y_{0} \in C\left(f, x_{0}\right)$, the cluster set of $x_{0}$. We show that $y_{0} \in\left(\partial G_{+} \cup \partial G_{-}\right) \times \mathbb{R}$. If $y_{0} \neq \infty$, then $\lim _{x \rightarrow x_{0}} f(x)=y_{0}$ by [V1, $\S 17.13$, 14]. On the other hand, $x_{0} \in C\left(f^{-1}, y_{0}\right)$ and $\lim _{y \rightarrow y_{0}} f^{-1}(y)=x_{0}$ by the same theorem. Therefore $f$ extends to a homeomorphism $f:(G \times \mathbb{R}) \cup\left\{x_{0}\right\} \rightarrow$ $(G \times \mathbb{R}) \cup\left\{y_{0}\right\}$ and $y_{0} \in\left(\partial G_{+} \cup \partial G_{-}\right) \times \mathbb{R}$, by [V1, $\left.\S 17\right]$.

If $y_{0}=\infty$, then the above argument shows that $C\left(f, x_{0}\right)=\{\infty\}$, and for $w \in G$ we have either

$$
f^{-1}(w, t) \rightarrow x_{0} \quad \text { as } t \rightarrow+\infty, \quad \text { or } \quad f^{-1}(w, t) \rightarrow x_{0} \quad \text { as } t \rightarrow-\infty,
$$

because $G \times \mathbb{R}$ is quasiconformally collared at $x_{0}$ and it is 2-connected at $\infty$. Hence a connected neighborhood of $\infty$ in $G \times \mathbb{R}$ is mapped by $f$ onto a neighborhood of $x_{0}$ in $G \times \mathbb{R}$. This is impossible because $G \times \mathbb{R}$ is quasiconformally collared at $x_{0}$ but not at $\infty$. Thus $y_{0} \neq \infty$ and we are back to the previous case.

We have shown that, for each $x_{0} \in\left(\partial G_{+} \cup \partial G_{-}\right) \times \mathbb{R}$,

$$
C\left(f, x_{0}\right)=\left\{y_{0}\right\} \subset\left(\partial G_{+} \cup \partial G_{-}\right) \times \mathbb{R}
$$

and that $C\left(f^{-1}, y_{0}\right)=\left\{x_{0}\right\}$. Therefore $f$ has an extension to an embedding, which we again denote by $f$, of $\left(\partial G_{+} \cup \partial G_{-}\right) \times \mathbb{R}$ so that

$$
f\left(\left(\partial G_{+} \cup \partial G_{-}\right) \times \mathbb{R}\right) \subset\left(\partial G_{+} \cup \partial G_{-}\right) \times \mathbb{R} .
$$

We show that the above inclusion holds with equality. Choose $y \in$ $\left(\partial G_{+} \cup \partial G_{-}\right) \times \mathbb{R}$ and let $\{x\}=C\left(f^{-1}, y\right)$. Then $C(f, x)=\{y\}$ and $y \in$ $f\left(\left(\partial G_{+} \cup \partial G_{-}\right)\right) \times \mathbb{R}$, whence

$$
f\left(\left(\partial G_{+} \cup \partial G_{-}\right) \times \mathbb{R}\right)=\left(\partial G_{+} \cup \partial G_{-}\right) \times \mathbb{R} .
$$

In addition, $f\left(\partial G_{+} \times \mathbb{R}\right)=\partial G_{+} \times \mathbb{R}$ or $f\left(\partial G_{+} \times \mathbb{R}\right)=\partial G_{-} \times \mathbb{R}$. Indeed if $x \in \partial G_{+} \times \mathbb{R}$ and if $f(x) \in \partial G_{+} \times \mathbb{R}$, then each $x^{\prime} \in \partial G_{+} \times \mathbb{R}$ can be joined to $x$ by an arc $\beta$ in $\partial G_{+} \times \mathbb{R}$. Since $\beta \cap L_{i}=\varnothing, f(\beta) \cap L_{i}=\varnothing$ for $i=1,2$. 
Hence $f(\beta) \subset \partial G_{+} \times \mathbb{R}$ and thus $f\left(x^{\prime}\right) \in \partial G_{+} \times \mathbb{R}$. Therefore we may assume that $f\left(\partial G_{+} \times \mathbb{R}\right)=\partial G_{+} \times \mathbb{R}$ and $f\left(\partial G_{-} \times \mathbb{R}\right)=\partial G_{-} \times \mathbb{R}$.

(b) Construction of the extension. For $x=(u, v, t), z=(u, v)$,

$$
h_{1}(x)= \begin{cases}\left(u, \frac{1}{2}(v-g(u)), t\right) & \text { if } u \in[0,1], \\ x & \text { otherwise }\end{cases}
$$

is 2-bilipschitz, and hence is a 16-quasiconformal self-map of $\mathbb{R}^{3}$ and

$$
h_{1}(G \times \mathbb{R})=G_{-} \times \mathbb{R}, \quad h_{1}\left(\partial G_{+} \times \mathbb{R}\right)=F, \quad h_{1}\left(\partial G_{-} \times \mathbb{R}\right)=\partial G_{-} \times \mathbb{R},
$$

where $F=\{(u, 0, t): 0 \leq u \leq 1\}$.

Hence $f_{1}=h_{1} \circ f \circ h_{1}^{-1}$ is a quasiconformal self-map of $G_{-} \times \mathbb{R}$ and induces a homeomorphism $h_{2}$ on $F$. By [V1, Theorem 35.3] $h_{2}$ is a quasiconformal self-map of $F$. By repeated use of the reflection principle we extend $h_{2}$ to a quasiconformal self-map $h_{3}$ of $H^{2}=\{(u, 0, t): u, t \in \mathbb{R}\}$. By [A], $h_{3}$ extends to a quasiconformal map $h_{4}: H^{3} \rightarrow H^{3}$, where $H^{3}=\{(u, v, t): v>0\}$. Hence

$$
f_{2}(x)= \begin{cases}f_{1}(x) & \text { if } x \in F \cup\left(G_{-} \times \mathbb{R}\right), \\ h_{4}(x) & \text { if } x \in H^{3}\end{cases}
$$

is a quasiconformal self-map of $\left(G_{-} \times \mathbb{R}\right) \cup F \cup H^{3}$, by [V1, 335.1$]$. Next

$$
h_{5}(x)= \begin{cases}x+g(u) e_{2} & u \in[0,1], \\ x & \text { otherwise }\end{cases}
$$

is a 2-bilipschitz self-map of $\mathbb{R}^{3}$. In addition, $h_{5}\left(G_{-} \times \mathbb{R}\right) \cup\left(F \cup H^{3}\right)=H^{3}$, and hence $f_{3}=h_{5} \circ f_{2} \circ h_{5}^{-1}$ is a quasiconformal self-map of $H^{3}$. By reflection, we extend $f_{3}$ to a quasiconformal self-map $f_{4}$ of $\mathbb{R}^{3}$, and the desired extension is

$$
\tilde{f}=h_{1}^{-1} \circ h_{5}^{-1} \circ f_{4} \circ h_{5} \circ h_{1} \text {. }
$$

Let $\phi$ be a Möbius map of $\overline{\mathbb{R}}^{3}$ so that $\phi(\infty)=0$ and $\phi^{-1}(\infty) \notin \bar{D}$. Then $D^{\prime}=\phi(D)$ is not quasiconvex. Thus by [K, Theorem 5.8] and by Möbius invariance of the quasiconformal extension property we have the following result.

5.3. Theorem. The domain $D^{\prime}$ has the quasiconformal but not the Sobolev extension property.

\section{AN EXTENSION THEOREM FOR CYLINDRICAL DOMAINS}

6.1. Theorem. Suppose that $G \subset \mathbb{R}^{2}$ is a simply connected domain and that $D=G \times \mathbb{R}$ is $K$-quasiconformally equivalent to $B^{3}$. Then the following are equivalent.

(a) $D$ is a quasiball.

(b) $D$ has the extension property for quasiconformal maps.

(c) D satisfies the double-quasiball condition.

Proof. (a) $\Rightarrow(\mathrm{b})$ : If $D$ is a quasiball, there is a quasiconformal self-map $\psi$ of $\overline{\mathbb{R}}^{3}$ so that $\psi\left(B^{3}\right)=D$. Let $f$ be a quasiconformal self-map of $D$. Then $f_{1}=\psi^{-1} \circ f \circ \psi$ is a quasiconformal self-map of $B^{3}$ which we can extend to a quasiconformal self-map $f_{2}$ of $\overline{\mathbb{R}}^{3}$ by reflection and $F=\psi \circ f_{2} \circ \psi^{-1}$ is the desired extension. 
(b) $\Rightarrow$ (c): This is proved in Theorem 3.2.

(c) $\Rightarrow($ a) : We first show that $\infty \in \partial G$. For if $G$ is bounded, then there is a quasiball (and hence $b$-uniform) $U \subset D$ so that $\infty \in \partial U$. If $x \in U$, then there is a $b$-uniform arc $\beta$ joining $x$ to $\infty$ in $Q \subset D$. Choose $x^{\prime} \in \beta$ so that $\left|x-x^{\prime}\right|>b \operatorname{diam} G$. Then $\operatorname{dist}\left(x^{\prime}, \partial D\right) \geq(1 / b)\left|x-x^{\prime}\right|>\operatorname{diam} G$, which is impossible.

Next, since $G \times \mathbb{R}$ is $K$-quasiconformally equivalent to $B^{3}, G$ is a $c$-inner chordarc domain, $c=c(K)$, by [V3] and $G$ is finitely connected on the boundary. By the double-quasiball assumption $\partial D=\partial D^{*}, \partial G=\partial G^{*}$, and $G$ is a Jordan domain.

We next show that $G$ is a quasidisk. Let $\gamma$ be a bounded arc in $\partial G$ from $z_{1}$ to $z_{2}$. If $\left\{\alpha_{j}\right\}$ are the components of $\left(z_{1}, z_{2}\right) \backslash \gamma$, and if $\gamma_{j}$ is the subarc of $\gamma$ having the same end points as $\alpha_{j}$, then $\operatorname{diam}(\gamma) \leq \sum \operatorname{diam}\left(\gamma_{j}\right)+\left|z_{1}-z_{2}\right|$. Hence to show that $G$ is a quasidisk it is enough to check that $\operatorname{diam}(\gamma) \leq c^{\prime}\left|z_{1}-z_{2}\right|$ for all $z_{1}$ and $z_{2}$ which are the end points of straight crosscuts of $G$ or $G^{*}$. We consider the latter case; the other case is similar.

Choose a point $z \in \gamma$ such that $\left|z_{j}-z\right| \geq(1 / 4) \operatorname{diam}(\gamma), j=1,2$. There is a $K^{\prime}$-quasiball (and hence $b$-uniform) $Q^{\prime} \subset D^{*}$ containing $x=(z, 0)$ in its boundary so that $\operatorname{diam}\left(Q^{\prime}\right)>4 b \operatorname{diam}(\gamma)$, by the hypothesis. Choose $x^{\prime} \in Q^{\prime}$ so that $\left|x-x^{\prime}\right| \geq 2 b \operatorname{diam}(\gamma)$, and let $\beta$ be a $b$-uniform arc in $Q^{\prime}$ between $x$ and $x^{\prime}$. If $x^{\prime \prime}=\left(z^{\prime \prime}, t^{\prime \prime}\right)$ is the midpoint of $\beta$, then

$$
\operatorname{dist}\left(z^{\prime \prime}, \partial G\right) \geq \operatorname{dist}\left(x^{\prime \prime}, \partial Q^{\prime}\right) \geq \frac{1}{2 b} l(\beta) \geq \operatorname{diam}(\gamma) .
$$

As a result $\beta \cap\left(\left[z_{1}, z_{2}\right] \times \mathbb{R}\right) \neq \varnothing$ and we can choose a point $y=(w, t) \in \beta$ so that $w \in\left[z_{1}, z_{2}\right]$. Thus

$$
\left|z_{1}-z_{2}\right| \geq \operatorname{dist}(w, \partial G) \leq \operatorname{dist}\left(y, \partial Q^{\prime}\right) \geq \frac{1}{b}|y-x| \geq \frac{1}{4 b} \operatorname{diam}(\gamma),
$$

as desired.

We now show that $G$ is a chordarc domain. Let $\gamma$ be a bounded arc in $\partial G$ from $z_{1}$ to $z_{2}$. Since $G$ is a quasidisk, it is $b^{\prime}$-uniform and there is an arc $\beta$ joining $z_{1}$ and $z_{2}$ in $G$ so that $l(\beta) \leq b^{\prime}\left|z_{1}-z_{2}\right|$. Since $G$ is a $c$-inner chordarc,

$$
l(\gamma) \leq c \lambda\left(z_{1}, z_{2}\right) \leq c l(\beta) \leq c b^{\prime}\left|z_{1}-z_{2}\right|
$$

and $G$ is a chordarc domain.

Hence $G^{*}$ is also an inner chordarc domain and $G^{*} \times \mathbb{R}$ is quasiconformally equivalent to $B^{3}$. Finally, both $D$ and $D^{*}$ are quasiconformally equivalent to $B^{3}$, and $D$ is a quasiball by $[\mathrm{G} 1]$.

\section{REFERENCES}

[A] L. V. Ahlfors, Extension of quasiconformal mappings from two to three dimensions, Proc. Nat. Acad. Sci. U.S.A. 51 (1964), 768-771.

[G1] F. W. Gehring, Extension of quasiconformal mappings in 3-space, J. Analyse Math. 14 (1965), 171-182.

[G2] M. Ghamsari, Inner chordarc domains (to appear).

[GH] F. W. Gehring and K. Hag, Remarks on uniform and quasiconformal extension domains, Complex Variables 9 (1987), 175-188. 
[GM] F. W. Gehring and O. Martio, Quasiextremal distance domains and extension of quasiconformal mappings, J. Analyse Math. 45 (1985), 203-219.

[GR] V. M. Goldstein and Y. G. Reshetnyak, Quasiconformal mappings and Sobolev spaces, Kluwer, Dordrecht, 1990.

[GV] F. W. Gehring and J. Väisälä, The coefficients of quasiconformality in space, Acta Math. 114 (1965), 1-70.

[J] P. Jones, Quasiconformal mappings and extendability of functions in Sobolev spaces, Acta Math. 147 (1981), 71-88.

[K] P. Koskela, Capacity extension domains, Ann. Acad. Sci. Fenn. Ser. AI 73 (1990).

[N1] R. Näkki, Continuous boundary extension of quasiconformal mappings, Ann. Acad. Sci. Fenn. Ser. AI 511 (1972), 1-10.

[N2] _ Prime ends, J. Analyse Math. 35 (1979), 13-40.

[TV1] P. Tukia and J. Väisälä, Quasisymmetric embeddings of metric spaces, Ann. Acad. Sci. Fenn. Ser. AI 5 (1980), 97-114.

[TV2] _ Bilipschitz extension of homeomorphisms which have quasiconformal extension, Math. Ann. 269 (1984), 561-572.

[V1] J. Väisälä, Lectures on quasiconformal mappings, Springer-Verlag, Berlin and New York, 1971.

[V2] _-, Quasisymmetric embeddings in euclidean spaces, Trans. Amer. Math. Soc. 264 (1981), 191-204.

[V3] _-, Quasiconformal maps of cylindrical domains, Acta Math. 162 (1989), 201-225.

[V4] _ Invariants for quasisymmetric, quasimöbius and bilipschitz maps, J. Analyse Math. 50 (1988), 201-223.

Department of Mathematics, University of Cincinnati, Cincinnati, Ohio 45221-0025

Current address: Department of Mathematics, Christopher Newport University, Newport News, Virginia 23606 